\title{
Intra- and inter-muscular differences in the cross-sectional area of the quadriceps muscles assessed by extended field-of-view ultrasonography
}

\author{
Chrysostomos Sahinis ${ }^{1}$, Eleftherios Kellis ${ }^{1}$, Nikiforos Galanis ${ }^{2}$, Konstantinos Dafkou ${ }^{1}$, \\ Athanasios Ellinoudis ${ }^{1}$
}

${ }^{1}$ Laboratory of Neuromechanics, Department of Physical Education and Sport Sciences at Serres, ${ }^{2}$ Medical School, Aristotle University of Thessaloniki, Thessaloniki, Greece

\begin{abstract}
Aim: To examine the inter- and intra-muscular differences in the anatomical cross-sectional area (CSA) of the quadricep muscles, using extended - field of view (EFOV) ultrasonography (US). Material and methods: Panoramic transverse US images of the thigh were acquired from 10 young participants at five different locations across the thigh, in two sessions, spaced a week apart. The CSA of the vastus medialis (VM), rectus femoris (RF), vastus intermedius (VI), vastus lateralis (VL) and tensor vastus intermedius (TVI) was quantified. Results: The intraclass correlation coefficients ranged from 0.75 to 0.97 and the standard error of measurement ranged from $0.78 \%$ to $6.61 \%$, indicating high test-retest reliability. Analysis of the variance indicated that among the 5 quadriceps muscles the VL and the RF displayed the greater CSA proximally, the VI medially and the VM distally across the thigh $(\mathrm{p}<0.05)$. No differences in the quadriceps CSA measured with and without including the TVI were found $(p>0.05)$. Conclusions: The EFOV US technique provides transverse scans of the quadriceps muscle in vivo and allowed a reliable and non-invasive determination of CSA at a low cost. Evaluation of CSA along the thigh largely depends on the measurement site. Future studies that examine the quadriceps CSA using EFOV after any form of intervention should consider changes of at least $6.5 \%$ as meaningful.
\end{abstract}

Keywords: ultrasound; extended - field of view; quadriceps; reliability

\section{Introduction}

The development of the extended field-of-view (EFOV) ultrasonography (US) enabled the capture of high-quality two-dimensional panoramic images of the underlying tissues up to $60 \mathrm{~cm}$ in length, thus allowing the assessment of large muscle group morphology. For this reason, many studies have examined muscle morphology using EFOV US [1-10].

Received 05.11.2019 Accepted 12.01.2020

Med Ultrason

2020, Vol. 22, No 2, 152-158

Corresponding author: Eleftherios Kellis, Ph.D.

Professor in Sports Kinesiology Laboratory of Neuromechanics, Aristotle University of Thessaloniki TEFAA Serres Serres, 62100 Greece Email: ekellis@phed-sr.auth.gr
An important determinant of muscle strength is the cross-sectional area (CSA) of the muscle. Owing to a limited field of view, quantification of CSA of large muscles, such as the quadriceps, using the conventional US is difficult $[2,3,11]$. Research studies have reported that measurement of the CSA of the quadriceps using EFOV US displayed good agreement with MRI [1,3] and CT [2] and a high intra- [2,4] and inter-examiner [3] reliability, with intraclass correlation coefficient (ICC) values being higher than 0.87 . Nevertheless, none of those studies have examined the reliability of CSA of individual quadricep muscles.

The quadriceps femoris is composed of the rectus femoris (RF), vastus medialis (VM), vastus intermedius (VI) and vastus lateralis (VL) [12]. A few studies have examined the anatomical CSA of the VM [2] and RF [3] using the EFOV technique but none have examined the 
VI CSA. In addition, a fifth head, the tensor of vastus intermedius (TVI) $[13,14]$ is often described and it is located between the VL and the VM [13,14]. Although anatomical descriptions of the TVI in cadavers [13] and US [14] have been reported, the contribution of this muscle to whole quadriceps muscle function is unclear.

The morphology of the quadriceps changes along its length [2,12,15-17]. It has been reported [15] that the CSA of the quadriceps femoris was maximal near the middle of the thigh with the greatest value presented by the VL [16]. Maximal CSA was observed distally for the VM and proximally for the VL and VI [17]. To our knowledge, only Noorkoiv et al [2] used EFOV US and they found that the CSA of the entire muscle group is greater proximally compared with distal locations.

Muscle atrophy is frequently observed in various clinical conditions such as spinal cord injury [18], chronic obstructive pulmonary disease [19], type 2 diabetes [20], cancer [21] and in elderly [22]. Therefore, assessment of CSA can be useful to quantify the degree of muscle atrophy and muscle function in clinical populations $[7,8,22-24]$ as well as to monitor changes in muscle size following various interventions $[8,25]$

Examination of the CSA along the length of the quadriceps is useful for the quantification of the regional mechanical properties of the muscle [26]. In addition, the precise functional contribution of the TVI to the whole quadriceps muscle group remains unclear. The use of EFOV overcomes the limitation of the conventional US systems thus allowing quantification of the anatomical CSA of individual components of the quadriceps muscle group. However, potential variations in the morphology of musculature along muscle length may influence the reliability of CSA measurements obtained using EFOV US.

The purpose of this study was, first, to compare the CSA of the 5 components of the quadriceps at various sections along the thigh, secondly, to examine the contribution of the TVI to the whole quadriceps group CSA along muscle length and, finally, to report on the reliability of EFOV US.

\section{Materials and methods}

\section{Participants}

Ten recreationally active young males and females [mean \pm standard deviation (SD) age 21.6 2.41 years; weight $80 \pm 5.17 \mathrm{~kg}$; height $182 \pm 7.21 \mathrm{~cm}$; femur length $49.15 \pm 3.62 \mathrm{~cm}$; distance between TVI and patella $31.6 \pm 3.49 \mathrm{~cm}$ ) participated in this study. They signed and completed a consent form, approved by the local university Ethics Committee. Participants were healthy and free of any lower limb injury.

\section{Design}

Quadriceps EFOV US scans were taken from the right, along the thigh on two occasions, spaced 5-7 days apart to establish the reliability of the measurements. Measurements were performed from the same investigator who had more than three years' experience with musculoskeletal US.

\section{Procedure}

The US images were obtained using a portable brightness mode (B-mode) imaging device (GE Logiq e, USA) and a multifrequency linear-array probe (12 L-RS, 5-13 MHz, 40.0-mm field-of-view).

The US measurements were performed with the subject lying in the supine position. The length of the right femur was measured as the distance between the greater trochanter (proximal) to the outer femoral condyle (distal) with an anthropometric measuring tape. To achieve acoustic coupling and to avoid any misinterpretation of the obtained images due to the pressure of the transducer, a water-based gel was applied between the skin and the transducer. First, a series of scans were performed in order to identify the approximate path of all muscles along the thigh. Special attention was given for the identification of the TVI which was identified only in transverse US images with a muscle belly identified proximally between the VL and the VI while the long distal tendon was visible approximately at the half length of the thigh [14]. Subsequently, starting from the proximal end, we divided the thigh in 5 sections at $40 \%$ (Proximal), 50\% (Proximal-Middle), 60\% (Middle), 70\% (Distal-Middle) and $80 \%$ (Distal) of femur length. For the separation of each section we used adhesive tape $2.5-\mathrm{cm}$ slices (fig 1). The placement of the tape created a path that the ultrasound probe could be guided through to obtain the panoramic image [3]. The distance between successive tapes was also measured to ensure that the placement of the adhesive tape was the same in the subsequent sessions.

For the acquisition of the images the probe was moved manually in the transverse axis at a slow and continuous rate from the lateral to the medial side of the quadriceps femoris. In every scan, a consistent minimum pressure was applied on the skin to avoid compression of the muscle (fig 1). If the quality of the image was unclear or a muscle was not fully depicted with identifiable borderlines, we repeated the measurement. A minimum of 5 scans in each site were taken to ensure acceptable image quality.

\section{Analysis}

The region of interest was carefully selected to exclude any surrounding bone or fascia. The contours of the RF, the VL, the VI, the VM and the TVI were first identi- 


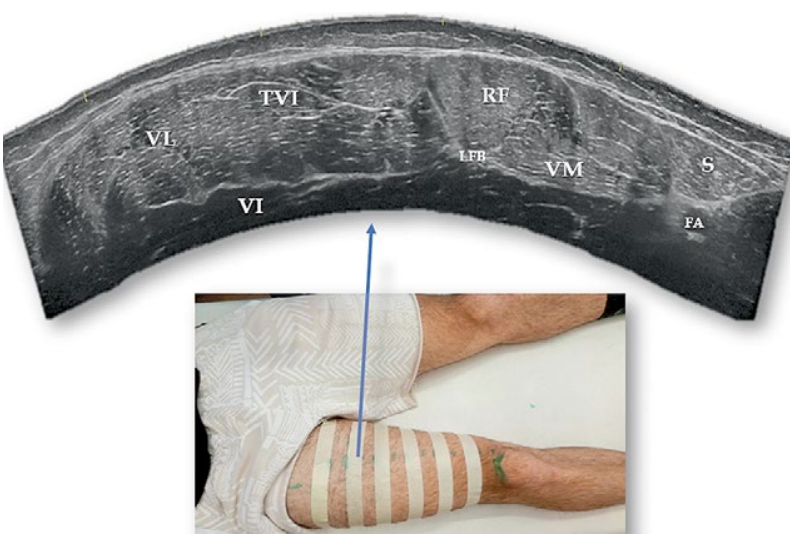

Fig 1. Upper image: Representative extended field of the view image of Rectus Femoris (RF), the Vastus Lateralis (VL), the Vastus Intermedius (VI), the Vastus Medialis (VM) and the Tensor of the Vastus Intermedius (TVI) obtained from the proximal-middle section. The sartorius (S) muscle, the lateral circumflex femoral descending branch (LFB) and the Femoral Artery (FA) are also identified. Lower image: Illustration of the ultrasound measurement paths (sections) using tapes.

fied on the captured US panoramic image by manually digitizing the images using image-based software (ImageJ version: 1.52a, National Institutes of Health, USA). After calibrating each image from pixels to $\mathrm{cm}$ using a known distance of $1 \mathrm{~cm} \mathrm{[27],} \mathrm{the} \mathrm{CSA} \mathrm{was} \mathrm{measured} \mathrm{us-}$ ing the polygon function of this software (fig 2).

Figure 3 presents the identified muscle contours per section. Particularly, all muscle CSAs were identified in the proximal and proximal-distal sections. The TVI contours were not clearly identifiable in the middle, distalmiddle and distal sections. Similarly, in the most distal section, the RF contours were not clearly visible.

To examine the influence of TVI on overall quadriceps muscle size, we calculated the quadriceps muscle CSA with and without including the TVI CSA in the proximal and proximal-middle sections.

\section{Statistical analysis}

\section{Reliability}

The $\mathrm{ICC}_{3,3}$ with $95 \%$ confidence interval (CI: 95\%) based on the average of 3 measurements per measurement site was calculated. An ICC value $\leq 0.50$ was considered as low, 0.50 to 0.75 was considered as moderate, $\geq 0.75$ was considered as good and $\geq 0.90$ was considered as excellent. Bland-Altman analysis was applied which includes the Bias and the limits of agreement (LoA) [28] between measurement sessions. The standard error of measurement (SEM) was also calculated using the formula: $S E M=S D \sqrt{1-I C C}$ where $\mathrm{SD}$ is the standard deviation of the differences between test and retest values. The analysis was performed using SPSS software (version 24, IBM, Chicago).

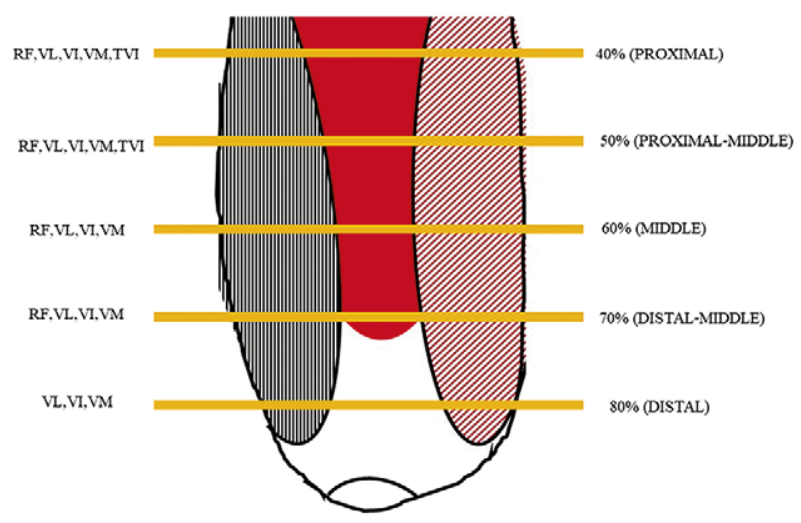

Fig 2. Illustration of the identified muscles per section. RF $=$ Rectus Femoris, VL = Vastus Lateralis, VI = Vastus Intermedius, $\mathrm{VM}=$ Vastus Medialis and TVI $=$ Tensor of the Vastus Intermedius.

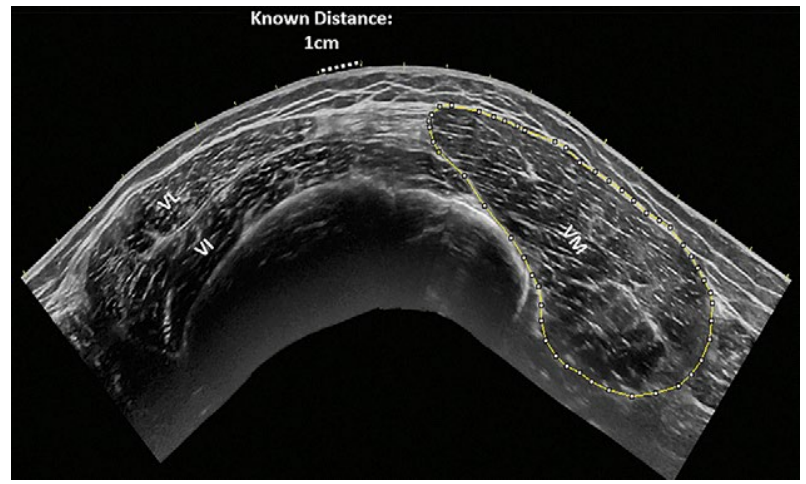

Fig 3. Example of transverse plane extended field of view ultrasound image of vastus lateralis (VL), vastus intermedius (VI) and vastus medialis (VM) muscles. Measurement of muscle cross-sectional area of each muscle (the VM in this image) was performed by manually tracing the border of the muscle using the polygon selection function in ImageJ software. Calibration of each image was performed individually using a known distance of $1 \mathrm{~cm}$.

\section{Inter-muscular comparisons}

Differences between muscles were examined using a two-way analysis of variance (ANOVA). A two-way ANOVA was used to examine the differences in CSA between five muscles (VM, RF, VL, VM and TV) in two (Proximal and Proximal-middle) sections. For middle and proximal-distal sections, a separate two-way ANOVA was used to examine differences between 4 muscles (VM, RF, VL, VI). Finally, a one-way ANOVA was used to examine differences in distal CSA values between 3 muscles (VM, VL, VI).

\section{Intra-muscular comparisons}

For each muscle, one-way ANOVA with repeated measures designs were applied to compare CSA values between different sections. 
Influence of TVI on total quadriceps CSA

A two-way ANOVA was implemented to examine the difference in total quadriceps CSA with and without including the TVI CSA in proximal and proximal-mid sections.

\section{Results}

\section{Reliability}

The results from the reliability analysis are presented in Table I. The ICC values ranged from 0.75 to 0.97 . The SEM values ranged from $0.009 \mathrm{~cm}^{2}$ to $1.218 \mathrm{~cm}^{2}(0.78 \%$ to $6.61 \%$ of the measured CSA). Overall the systematic error (bias) ranged from $-0.474 \mathrm{~cm}^{2}$ to $0.654 \mathrm{~cm}^{2}$.

\section{Inter-muscular comparisons}

The mean group values of the CSA are presented in Table II. For the Proximal and Proximal-Middle sections, the ANOVA indicated that there was a section by muscle interaction effect $(p<0.05)$. Post-hoc Tukey tests indicated that in the Proximal section, the VL and TVI displayed the greater and lower CSA, respectively $(p<0.05)$. The VI showed greater CSA than the RF and VM while the RF CSA was greater than VM CSA. Similar differences were also found in the Proximal-Middle section values, with the exception of VL and VI which were not statistically significant $(\mathrm{p}>0.05)$.

For the Middle and Middle-Distal section data, there was a statistically significant section by muscle interaction effect on CSA ( $p<0.05)$. Post-hoc Tukey tests indicated that the VL CSA and VI CSA were greater than the $R F$ and VM values $(p>0.05)$. No other differences were found $(\mathrm{p}>0.05)$

The ANOVA also showed a significant difference in Distal CSA values $(\mathrm{p}<0.05)$. Post-Hoc Tukey tests, showed that the VM CSA was greater than VL and VI CSA values $(\mathrm{p}<0.05)$.

\section{Intra-muscular comparisons}

The group values of CSA of each muscle at different measurement sections are presented in Figure 4. The ANOVA results followed by post-hoc Tukey tests indicated that the RF CSA was lower in the Distal and DistalMiddle sections compared with the remainder sections $(\mathrm{p}<0.05)$. The Distal and Distal-Middle CSAs of the RF were also significantly different $(\mathrm{p}<0.05)$. The VL and VI CSA in the distal section were lower compared with all other section values $(\mathrm{p}<0.05)$. In contrast, the VM CSA recorded at Distal, Distal-Middle and Middle sec-

Table I. Reliability values of each muscle of quadriceps femoris for the Proximal, the Proximal-middle, the Middle, the Distal-middle and the Distal site across the femur length.

\begin{tabular}{|c|c|c|c|c|c|c|}
\hline Measurements sites & Test $\left(\mathrm{cm}^{2}\right)$ & R-test $\left(\mathrm{cm}^{2}\right)$ & $\mathrm{ICC}_{2,1}$ & SEM & Bias \pm Lower LoA & Upper LoA \\
\hline \multicolumn{7}{|l|}{ Proximal Site } \\
\hline Rectus Femoris & $12.93 \pm 2.64$ & $12.76 \pm 2.39$ & 0.97 & 0.282 & $0.173 \pm-0.410$ & 0.757 \\
\hline Vastus lateralis & $25.38 \pm 4.73$ & $26.19 \pm 5.47$ & 0.95 & 0.410 & $-0.077 \pm-1.544$ & 1.390 \\
\hline Vastus Intermedius & $20.25 \pm 3.64$ & $20.54 \pm 3.43$ & 0.92 & 0.764 & $0.288 \pm-1.078$ & 1.656 \\
\hline Vastus Medialis & $4.53 \pm 1.00$ & $4.48 \pm 0.70$ & 0.75 & 0.252 & $0.048 \pm-0.312$ & 0.409 \\
\hline Tensor of vastus intermedius & $1.31 \pm 0.09$ & $1.29 \pm 0.01$ & 0.92 & 0.014 & $0.019 \pm-0.018$ & 0.057 \\
\hline \multicolumn{7}{|l|}{ Proximal-Middle Site } \\
\hline Rectus Femoris & $11.86 \pm 1.56$ & $11.74 \pm 1.93$ & 0.88 & 0.388 & $0.121 \pm-0.681$ & 0.924 \\
\hline Vastus lateralis & $25.15 \pm 3.41$ & $24.87 \pm 5.34$ & 0.92 & 0.688 & $0.280 \pm-1.162$ & 2.022 \\
\hline Vastus Intermedius & $23.45 \pm 3.36$ & $22.8 \pm 2.79$ & 0.78 & 1.218 & $0.654 \pm-1.204$ & 2.513 \\
\hline Vastus Medialis & $6.14 \pm 1.64$ & $6.06 \pm 1.49$ & 0.86 & 0.406 & $0.088 \pm-0.689$ & 0.866 \\
\hline Tensor of vastus intermedius & $1.21 \pm 0.10$ & $1.22 \pm 0.07$ & 0.94 & 0.009 & $0.014 \pm-0.014$ & 0.043 \\
\hline \multicolumn{7}{|l|}{ Middle Site } \\
\hline Rectus Femoris & $8.33 \pm 2.92$ & $8.30 \pm 1.83$ & 0.88 & 0.550 & $0.032 \pm-1.105$ & 1.169 \\
\hline Vastus lateralis & $22.56 \pm 3.98$ & $22.68 \pm 3.70$ & 0.96 & 0.296 & $-0.112 \pm-1.173$ & 0.949 \\
\hline Vastus intermedius & $22.09 \pm 4.03$ & $21.61 \pm 3.36$ & 0.84 & 1.074 & $-0.474 \pm-2.396$ & 1.446 \\
\hline Vastus medialis & $9.80 \pm 1.45$ & $10.02 \pm 1.85$ & 0.84 & 0.488 & $-0.218 \pm-1.092$ & 0.654 \\
\hline \multicolumn{7}{|l|}{ Distal-Middle Site } \\
\hline Rectus Femoris & $4.95 \pm 1.93$ & $4.68 \pm 1.65$ & 0.85 & 0.462 & $0.267 \pm-0.730$ & 1.266 \\
\hline Vastus lateralis & $20.30 \pm 4.75$ & $20.28 \pm 4.36$ & 0.93 & 0.587 & $0.024 \pm-1.564$ & 1.613 \\
\hline Vastus intermedius & $20.27 \pm 2.96$ & $20.47 \pm 3.13$ & 0.86 & 0.782 & $-0.200 \pm-1.695$ & 1.294 \\
\hline Vastus medialis & $14.37 \pm 2.62$ & $14.68 \pm 3.54$ & 0.90 & 0.586 & $0.317 \pm-1.008$ & 1.644 \\
\hline \multicolumn{7}{|l|}{ Distal Site } \\
\hline Vastus lateralis & $11.61 \pm 3.06$ & $11.31 \pm 2.71$ & 0.91 & 0.490 & $0.305 \pm-0.864$ & 1.474 \\
\hline Vastus intermedius & $11.92 \pm 3.24$ & $11.62 \pm 2.75$ & 0.90 & 0.568 & $0.303 \pm-0.981$ & 1.588 \\
\hline Vastus medialis & $25.08 \pm 3.77$ & $25.04 \pm 4.77$ & 0.93 & 0.576 & $0.045 \pm-1.512$ & 1.602 \\
\hline
\end{tabular}

The results are expressed as mean \pm standard deviation. $\mathrm{ICC}=$ Intraclass Correlation Coefficient, $\mathrm{SEM}=$ standard error of measurement, Bias \pm LoA $=95 \%$ Limits of agreement. 
156 Chrysostomos Sahinis et al EFOV US: intra-and inter-muscular differences in the cross-sectional area of the quadriceps muscles

Table II. Mean cross-sectional area of each individual muscle at each measurement section

\begin{tabular}{llllll}
\hline \multirow{2}{*}{ Muscle } & \multicolumn{2}{l}{ Measurement section } & & & \\
\cline { 2 - 6 } & Proximal & Proximal-Middle & Middle & Distal-Middle & Distal \\
\hline Vastus lateralis (VL) & $25.38 \pm 4.73$ & $25.15 \pm 3.41$ & $22.56 \pm 3.98$ & $20.30 \pm 4.75$ & $11.61 \pm 3.06$ \\
Vastus intermedius (VI) & $20.25 \pm 3.64 \#$ & $23.45 \pm 3.36$ & $22.09 \pm 4.03$ & $20.27 \pm 2.96$ & $11.92 \pm 3.24$ \\
Rectus femoris (RF) & $12.93 \pm 2.64^{* \wedge}$ & $11.86 \pm 1.56^{* \wedge}$ & $8.33 \pm 2.92^{*}$ & $4.95 \pm 1.93^{* \wedge}$ & - \\
Vastus medialis (VM) & $4.53 \pm 1.00^{*}$ & $6.14 \pm 1.64^{*}$ & $9.80 \pm 1.45^{*}$ & $14.37 \pm 2.62^{*}$ & $25.08 \pm 3.77^{*}$ \\
Tensor of vastus intermedius (TVI) & $1.31 \pm 0.09^{* \wedge} \#$ & $1.21 \pm 0.10^{* \wedge}$ & - & - & - \\
\hline
\end{tabular}

The results are expressed as mean \pm standard deviation. ${ }^{*}=$ significantly different than $\mathrm{VL}$ and $\mathrm{VI} ; \#=$ different than $\mathrm{VL} ;{ }^{\wedge}=$ different than $\mathrm{VM}$; \# = different than RF

tions were greater than the remainder sections $(\mathrm{p}<0.05)$. In addition, CSA values differed between distal, distalmiddle and middle sections $(\mathrm{p}<0.05)$. As for the TVI CSA, no difference was found between the Proximal and Proximal-Middle sections ( $\mathrm{p}>0.05$ ).

\section{Influence of the TVI on quadriceps CSA}

The sum of the quadriceps CSA in the Proximal section was $63.09 \pm 12.01 \mathrm{~cm}^{2}$ and $64.4 \pm 12.10 \mathrm{~cm}^{2}$, with and without including the TVI CSA, respectively. The corresponding values in the Proximal-middle section were $66.6 \pm 9.97 \mathrm{~cm}^{2}$ and $67.81 \pm 10.07 \mathrm{~cm}^{2}$, respectively. The ANOVA indicated a non-statistically significant difference in quadriceps CSA measured with and without including the TVI $(\mathrm{p}>0.05)$.

\section{Discussion}

The main findings of this study were that anatomic CSA varied along the quadriceps muscle length and the TVI CSA did not influence the whole quadriceps group CSA. Further, EFOV US assessment of anatomical CSA of the quadricep muscles at different sections displays high reliability.

The values of the CSA of the muscles of the quadriceps are in line with previous studies which also applied the EFOV technique [1-5,10,29]. For example, studies have reported that the VL CSA $[1,3,5,29]$ ranges between $19.8 \pm 1.9 \mathrm{~cm}^{2}$ and $34.75 \pm 8 \mathrm{~cm}^{2}$, which is very similar to our values. Furthermore, Noorkoiv et al [2] reported that the CSA of VM was $27.4 \pm 7.2 \mathrm{~cm}^{2}$ which was observed distally in this study. Finally, the RF showed lower CSA values than the VL and VM, while its value was greater compared with that reported by Scot et al [3] $(6 \pm 2.2$ $\mathrm{cm}^{2}$ ), possibly because Scott et al [3] reported the average CSA of all sections and not the CSA values per section. Finally, no studies have examined the CSA of the VI and the TVI muscle using EFOV US.

The CSA of each component of the quadriceps femoris showed a heterogeneity in the separate measurement sites across the thigh. Each muscle displayed the

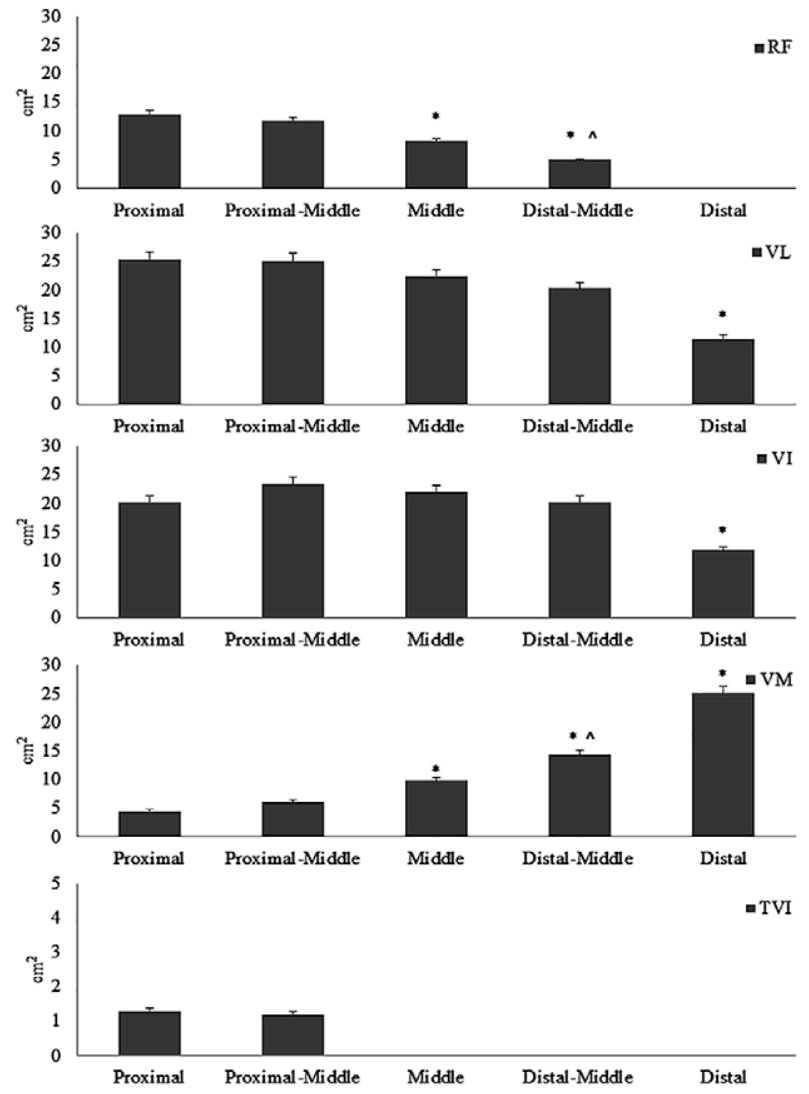

Fig 4. Mean cross-sectional group values of the Rectus Femoris (RF), the Vastus Lateralis (VL), the Vastus Intermedius (VI) and the Vastus Medialis (VM) and Tensor of the vastus intermedius (TVI) in different sections (Error bars indicate standard deviation, * significantly different compared with all other sections; ${ }^{\wedge}$ significantly different compared with Middle and Distal sections, $\mathrm{p}<0.05)$.

maximum CSA in a different measurement location. The variability of muscle architecture is not an uncommon phenomenon for the quadriceps femoris musculature $[12,15,30]$. For instance, in our study, the VM CSA was greater in the distal section which agrees with previous studies $[15,17]$. Similarly, previous research has shown that the VL and the RF presented the greater CSA proxi- 
mally $[15,16]$, which has also been observed in the study. In contrast to the other muscles, the VI had very similar CSA from proximal to middle-distal sections and decreases distally which agrees with previous studies $[12,15,16]$. From the above findings, it appears that the assessment of the quadriceps CSA using EFOV US largely depends on the location of the measurement. Evaluation of regional atrophy due to injury of a specific individual muscle or other pathological condition may provide additional information regarding the functional and force-generation capacity of each muscle.

We are aware of only a few studies to date that have provided anatomical descriptions of the TVI musculature using diagnostic imaging techniques [14,31]. The TVI CSA was approximately four to 24 times lower compared with the CSA of the remainder muscles. This is not surprising as the TVI is a small muscle entity with the main bulk being traced proximally, only in the transverse direction [14]. The precise role of this specific muscle is not clearly understood, as it has small muscle belly proximally and a very long distal tendon which attaches to the patella $[13,32]$. Assuming that the anatomic CSA is an indirect indicator of force generation capacity, our results indicate that the contribution of TVI to the whole quadriceps muscle force generation capacity is rather limited. Hence, examination of role of CSA for force generation capacity and function of the quadricep muscle group may well be performed without measuring the TVI CSA. However, when the aim is to quantify the CSA of each individual quadricep muscle component, especially the VM or the RF, failure to identify and exclude the TVI muscle from the CSA measurements may yield a significant overestimation of the CSA (up to approximately $25 \%$ ) of those muscles. Further studies are necessary to examine the role of TVI in the whole quadricep muscle function.

In our study, CSA measurements using EFOV US indicated high test-retest reliability with ICC values greater than 0.75 and a maximum SEM of $6.5 \%$. Melvin et al [4] also found that ICCs greater than 0.87 and a SEM of $2.12 \mathrm{~cm}^{2}$ for VL CSA. Others, however, reported ICC values $[2,3]$ greater than 0.95 which are greater than our results. The lowest ICCs reported in this study can be attributed to various factors. First, we measured the CSA of each individual muscle while previous studies $[2,3]$ examined the CSA of the whole quadriceps. It is possible that tracing the contours of each muscle component displays greater variability than the measurement of the contours of the whole muscle group. Second, we examined the CSA values in various locations along the thigh while previous studies [2,3] have measured the maximum CSA value. Hence, it is highly possible that minor variations in locating and placing the probe in repeated testing sessions resulted in greater variability of the obtained regional CSAs compared with the identification of the maximum CSA or the CSA at a specific location (applied by previous studies [2,3]). Collectively, the present results extend previous observations and indicate that EFOV US is a reliable method for the measurement of CSA of each individual quadriceps muscle CSA in vivo.

There are some limitations in this study. Firstly, the sample included only healthy individuals. It is possible that other groups, such as older or injured individuals, might have showed different CSA values as well as less reliable measures. Further, although the sample size is similar or greater than that employed by previous studies [2,3], a greater sample size would provide a better estimate of EFOV US measurement of CSA in the general population. Secondly, the examiner had a minimum of three-year experience with US. It is possible that less or more experienced examiners would produce different results. Thirdly, the inter-examiner reliability was not examined. Although the US measurement was performed with the muscle at rest and the US probe path is drawn on the skin, variability in probe placement and examiner experience cannot be excluded. Another limitation was that the activation of the quadriceps femoris during the test was not recorded. Although the participants were asked to relax as much as possible in each testing position, it is possible that muscle activation was not minimal. Finally, different equipment specifications such as the probe size and the panoramic mode algorithm implemented by the equipment manufacturer might influence the variability of the obtained measurements.

\section{Conclusion}

Application of the EFOV US showed proximo-distal variations in CSA of each individual quadricep muscle in vivo. The CSA of the TVI was the smallest compared to the other quadricep muscles, but it did not influence the whole quadriceps group CSA. The assessment of CSA of the quadriceps femoris using the EFOV technique, displayed a high reliability for all the measurement locations across the femur length.

Acknowledgments: We would like to thank the participants of the study for volunteering their time. No external financial support was received for this research.

\section{Conflict of interest: none}

\section{References}

1. Ahtiainen JP, Hoffren M, Hulmi JJ, et al. Panoramic ultrasonography is a valid method to measure changes in 
skeletal muscle cross-sectional area. Eur J Appl Physiol 2010;108:273-279.

2. Noorkoiv M, Nosaka K, Blazevich AJ. Assessment of quadriceps muscle cross-sectional area by ultrasound extended-field-of-view imaging. Eur J Appl Physiol 2010;109:631-639.

3. Scott JM, Martin DS, Ploutz-Snyder R, et al. Reliability and Validity of Panoramic Ultrasound for Muscle Quantification. Ultrasound Med Biol 2012;38:1656-1661.

4. Melvin MN, Smith-Ryan AE, Wingfield HL, Fultz SN, Roelofs EJ. Evaluation of Muscle Quality Reliability and Racial Differences in Body Composition of Overweight Individuals. Ultrasound Med Biol 2014;40:1973-1979.

5. Varanoske AN, Fukuda DH, Boone CH, Beyer KS, Stout JR, Hoffman JR. Scanning Plane Comparison of Ultrasound- Derived Morphological Characteristics of the Vastus Lateralis. Clin Anat 2017;30:533-542.

6. Trezise J, Collier N, Blazevich AJ. Anatomical and neuromuscular variables strongly predict maximum knee extension torque in healthy men. Eur J Appl Physiol 2016;116:1159-1177.

7. Ticinesi A, Narici MV, Lauretani F, et al. Assessing sarcopenia with vastus lateralis muscle ultrasound : an operative protocol. Aging Clin Exp Res 2018;30:1437-1443.

8. Scott JM, Martin DS, Ploutz-Snyder R, et al. Panoramic ultrasound: a novel and valid tool for monitoring change in muscle mass. J Cachexia Sarcopenia Muscle 2017;8:475-481.

9. Tomko PM, Muddle TW, Magrini MA, Colquhoun RJ, Luera MJ, Jenkins ND. Reliability and differences in quadriceps femoris muscle morphology using ultrasonography: The effects of body position and rest time. Ultrasound 2018;26:214-221.

10. Scanlon TC, Fragala MS, Stout JR, et al. Muscle architecture and strength: Adaptations to short-term resistance training in older adults. Muscle Nerve 2014;49:584-592.

11. Abe T, Loenneke JP, Thiebaud RS. Morphological and functional relationships with ultrasound measured muscle thickness of the lower extremity: a brief review. Ultrasound 2015;23:166-173.

12. Blazevich AJ, Cannavan D, Coleman DR, Horne S. Influence of concentric and eccentric resistance training on architectural adaptation in human quadriceps muscles. J Appl Physiol 2007;103:1565-1575.

13. Grob K, Ackland T, Kuster MS, Manestar M, Filgueira L. A newly discovered muscle: The tensor of the vastus intermedius. Clin Anat 2016;29:256-263.

14. Rajasekaran S, Hall MM. Sonographic Appearance of the Tensor of the Vastus Intermedius. PM R 2016;8:1020-1023.

15. Narici MV, Roi GS, Landoni L, Minetti AE, Cerretelli P. Changes in force, cross-sectional area and neural activation during strength training and detraining of the human quadriceps. Eur J Appl Physiol Occup Physiol 1989;59:310-319.

16. Behan FP, Maden-Wilkinson TM, Pain MTG, Folland JP. Sex differences in muscle morphology of the knee flexors and knee extensors. PLoS One 2018;13:e0190903.

17. Mersmann F, Bohm S, Schroll A, Boeth H, Duda G, Arampatzis A. Muscle shape consistency and muscle vol- ume prediction of thigh muscles. Scand J Med Sci Sports 2015;25:e208-e213.

18. Gorgey AS, Dudley GA. Skeletal muscle atrophy and increased intramuscular fat after incomplete spinal cord injury. Spinal Cord 2007;45:304-309.

19. Marquis K, Debigaré R, Lacasse Y, et al. Midthigh muscle cross-sectional area is a better predictor of mortality than body mass index in patients with chronic obstructive pulmonary disease. Am J Respir Crit Care Med 2002;166:809-813.

20. Wang T, Feng X, Zhou J, et al. Type 2 diabetes mellitus is associated with increased risks of sarcopenia and pre-sarcopenia in Chinese elderly. Sci Rep 2016;6:38937.

21. Miyamoto Y, Baba Y, Sakamoto Y, et al. Sarcopenia is a Negative Prognostic Factor After Curative Resection of Colorectal Cancer. Ann Surg Oncol 2015;22:2663-2668.

22. Chang KV, Wu WT, Huang KC, Jan WH, Han DS. Limb muscle quality and quantity in elderly adults with dynapenia but not sarcopenia: An ultrasound imaging study. Exp Gerontol 2018;108:54-61.

23. Seymour JM, Ward K, Sidhu PS, et al. Ultrasound measurement of rectus femoris cross-sectional area and the relationship with quadriceps strength in COPD. Thorax 2009;64:418-423.

24. Chang KV, Yang KC, Wu WT, Huang KC, Han DS. Association between metabolic syndrome and limb muscle quantity and quality in older adults: A pilot ultrasound study. Diabetes Metab Syndr Obes Targets Ther 2019;12:1821-1830.

25. Strasser EM, Draskovits T, Praschak M, Quittan M, Graf A. Association between ultrasound measurements of muscle thickness, pennation angle, echogenicity and skeletal muscle strength in the elderly. Age (Dordr) 2013;35:23772388 .

26. Mangine GT, Redd MJ, Gonzalez AM, et al. Resistance training does not induce uniform adaptations to quadriceps. PLoS One 2018; 13:e0198304.

27. Palmer TB, Akehi K, Thiele RM, Smith DB, Thompson BJ. Reliability of panoramic ultrasound imaging in simultaneously examining muscle size and quality of the hamstring muscles in young, healthy males and females. Ultrasound Med Biol 2015;41:675-684

28. Bland JM, Altman DG. Statistical methods for assessing agreement between two methods of clinical measurement. Lancet 1986;1:307-310.

29. Wells AJ, Fukuda DH, Hoffman JR, et al. Vastus lateralis exhibits non-homogenous adaptation to resistance training. Muscle Nerve 2014;50:785-793.

30. Ema R, Wakahara T, Miyamoto N, Kanehisa H, Kawakami Y. Inhomogeneous architectural changes of the quadriceps femoris induced by resistance training. Eur J Appl Physiol 2013;113:2691-2703.

31. Grob K, Manestar M, Gascho D, et al. Magnetic resonance imaging of the tensor vastus intermedius: A topographic study based on anatomical dissections. Clin Anat 2017;30:1096-1102.

32. Grob K, Manestar M, Filgueira L, Ackland T, Gilbey H, Kuster MS. New insight in the architecture of the quadriceps tendon. J Exp Orthop 2016;3:32. 